\title{
Avaliação física e química e aceitação pelo consumidor de laranjas 'Valência', produzidas sob sistemas de cultivo orgânico e convencional
}

\author{
Physical and chemical evaluation and consumer acceptance of 'Valencia' oranges grown under \\ organic and conventional production systems
}

\author{
Henrique Belmonte Petry ${ }^{I}$ Leonardo André Schneider II Jairo Camargo Silveira Júnior ${ }^{\text {II }}$ \\ Tainara de Moraes Crizel ${ }^{\text {III }}$ Simone Hickmann Flôres ${ }^{\text {III }}$ Sergio Francisco Schwarz ${ }^{\text {IV }}$
}

RESUMO

O objetivo foi verificar a influência do sistema de cultivo, orgânico e convencional, sobre as características físicas e químicas e sobre a aceitação pelo consumidor de laranjas 'Valência'(Citrus sinensis (L.) Osb.). Os pomares foram instalados no município de Montenegro, em julho de 2001, em espaçamento de 2,5mx5,0m, cada um possuindo uma área de 0,25ha. As avaliações foram realizadas a partir de amostras de frutos colhidas em setembro e outubro de 2011 e em outubro de 2012. Os parâmetros fisicos e químicos analisados nos frutos foram: teor de suco (TS), teor de sólidos solúveis (SS), acidez titulável (AT), relação SS/AT e índice de cor da casca. A avaliação da aceitação das laranjas foi realizada com avaliadores não treinados, e utilizando escala hedônica de nove pontos, que expressam o grau de gostar ou desgostar do produto. $O$ delineamento experimental foi completamente casualizado. Os frutos apresentaram parâmetros de qualidade adequados aos padrões internacionais e não diferiram entre si quanto as suas características fisicas e químicas na maioria dos atributos avaliados. A aceitação foi satisfatória em todos os atributos avaliados (todos superiores a 70\%). As diferenças observadas nos atributos físicos e químicos não foram percebidas pelos avaliadores, não havendo, portanto, distinção na aceitação dos frutos pelo consumidor entre os sistemas de cultivo.

Palavras-chave: Citrus sinensis, teste de aceitabilidade, qualidade dos frutos.

\section{ABSTRACT}

The objective of the research was to investigate the influence of cultivating systems (organic and conventional) on physical and chemical attributes, and consumer acceptance of 'Valencia' oranges (Citrus sinensis (L.) Osb.). The orchards were field planted in Montenegro, Brazil, in July 2001. Plants are spaced $2.5 \mathrm{~m}$ in the row by $5.0 \mathrm{~m}$ between rows, totaling 0.25 ha each orchard. Evaluations were made using fruit samples collected in September and October of 2011, and October of 2012. The physical and chemical attributes evaluated were: juice contents (TS), soluble solids (SS), titratable acidity (AT), ratio SS/AT and color index in the peel (ICC). The oranges' consumer acceptance was determined by untrained evaluators using a nine-point hedonic scale, where the degree in which they 'like' or 'dislike' the product is expressed. The experimental design was completely randomized. As results, oranges showed parameters of quality accordingly to international standards. The physical and chemical attributes were not different between conventional and organic systems for most attributes evaluated. Consumer acceptance was satisfactory in all attributes evaluated (all above 70\%). The differences observed on the physical and chemical attributes were not perceived by the evaluators; therefore, there is no distinction on the acceptability of the fruits by consumers despite of the cultivation system.

Key words: Citrus sinensis, acceptance test, fruit quality.

\section{INTRODUÇÃO}

A demanda por produtos orgânicos no Brasil e no mundo vem aumentando nos últimos anos, mesmo assim, em 2006, apenas $1,75 \%$ dos estabelecimentos eram produtores orgânicos (IBGE, 2013). No Rio Grande do Sul (RS) os citros são cultivados em diversos sistemas de produção. O mais usual é o convencional, o qual

\footnotetext{
'Empresa de Pesquisa Agrícola e Extensão Rural de Santa Catarina, Estação Experimental de Urussanga, Rd. SC 108, Km 353, Bairro Estação, 88840-000, Urussanga, SC, Brasil. E-mail: henriquepetry@epagri.sc.gov.br. Autor para correspondência.

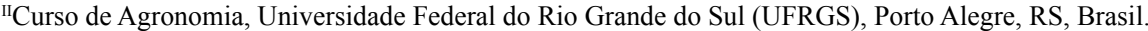

IIIPrograma de Pós-graduação (PPG) em Ciência e Tecnologia de Alimentos, UFRGS, Porto Alegre, RS, Brasil.

IVPrograma de Pós-graduação em Fitotecnia, UFRGS, Porto Alegre, RS, Brasil.
} 
não possui regras definidas ou legislação específica definida. São considerados pomares orgânicos os que atendem aos princípios estabelecidos pela Lei 10.831, especialmente no que tange à sustentabilidade ecológica e econômica. Produto orgânico, segundo a legislação vigente, é todo aquele em que se adotam técnicas específicas, mediante a otimização do uso dos recursos naturais e socioeconômicos disponíveis e o respeito à integridade cultural das comunidades rurais, tendo por objetivo a sustentabilidade econômica e ecológica, a maximização dos benefícios sociais, a minimização da dependência de energia não renovável (BRASIL, 2003).

A laranjeira 'Valência' é o principal cultivar copa de laranjas produzidas no Rio Grande do Sul, ocupando lugar de destaque entre os produtores, pela boa produtividade e adequado tamanho de frutos, que se prestam para frutas de mesa e à industrialização (PETRY et al., 2012). O consumidor brasileiro de frutas aponta a aparência do fruto como a característica mais relevante no momento da compra, embora a renda (poder de compra) e o preço dos produtos também afetam o consumo de frutas no Brasil (TEIXEIRA et al., 2006).

Já o mercado consumidor de frutas orgânicas é mais preocupado com as condições de produção, tanto em termos ambientais como sociais, do que com a aparência e sabor dos frutos (UNCTAD, 2013). Mesmo assim, não há como prescindir, em sistemas de agricultura orgânica de produção de frutas cítricas para consumo in natura, de alta qualidade, tanto na aparência quanto no sabor, além de que estas sejam alimentos seguros para o consumidor (AGOSTINI, 2007; PETRY et al., 2012).

Os citros, bem como outras frutas, variam na sua composição química, mesmo dentro da mesma variedade, dependendo de fatores edafoclimáticos, maturação e práticas agrícolas (PÉREZ-LÓPEZ et al., 2007), o que pode se refletir na qualidade e aceitação dos frutos. O mercado consumidor considera que produtos orgânicos são mais saudáveis, principalmente por considerar um risco a possível presença de resíduos de agrotóxicos nos frutos provenientes de pomares convencionais (SABA \& MESSINA, 2003). Porém, por falta de evidências científicas, ainda não se pode afirmar que os alimentos orgânicos são de fato mais saudáveis, saborosos e nutritivos que os alimentos produzidos convencionalmente (MAGKOS et al.,2006).

O objetivo deste trabalho foi verificar a influência do sistema de cultivo, orgânico e convencional, sobre as características físicas e químicas, bem como sobre a aceitação de laranjas 'Valência' por consumidores.

\section{MATERIAL E MÉTODOS}

A pesquisa foi realizada com laranjeiras 'Valência' (Citrus sinensis (L.) Osb.), enxertadas sobre Poncirus trifoliata Raf. em dois pomares experimentais, localizados no Centro de Formação da Emater/Ascar (2942'48”S; 51²8'33"W), situado no município de Montenegro-RS. O solo no local do experimento foi classificado como Argissolo Vermelho distrófico espessarênico (STRECK et al., 2008) e o clima subtropical úmido de verão quente do tipo Cfa (BERGAMASCHI et al., 2003). Os pomares foram implantados a $300 \mathrm{~m}$ de distância um do outro em agosto de 2001. Uma área foi conduzida, desde a implantação, no sistema de cultivo convencional e a outra em sistema orgânico. Em cada pomar, foram implantadas 147 plantas em espaçamento de $5,0 \mathrm{~m}$ entre linhas e $2,5 \mathrm{~m}$ entre plantas. Ambos os pomares foram protegidos dos ventos por uma linha de Pinus ellioti (linha externa), com espaçamento de $1,5 \mathrm{~m}$ entre plantas e uma linha com capim elefante (Pennisetum purpureum Schum.), 'Cameroon' (linha interna do quebravento), em torno de cada pomar.

O manejo do pomar convencional consistiu, basicamente, da utilização de adubos minerais (NPK) duas vezes ao ano (setembro e maio) e de calcário (em maio 3,6kg planta ${ }^{-1}$ PRNT 60\%); aplicação de herbicidas em área total em outubro e fevereiro (glifosato - $4 \mathrm{~L} \mathrm{ha}^{-1}$ ); aplicações de fungicidas (fosfito de potássio $0,25 \%$ em novembro e fevereiro; Cuprogarb ${ }^{\circledR} 0,3 \%$ em setembro, outubro, novembro, janeiro e março; Derosal $^{\circledR} 0,1 \%$, associado ao óleo mineral $0,5 \%$ na metade do desenvolvimento floral, Folicur ${ }^{\circledR}$ $0,15 \%$, no início da abertura dos botões florais e Dithane ${ }^{\circledR} 0,25 \%$, associado ao Cuprogarb ${ }^{\circledR} 0,3 \%$ ) e inseticidas (óleo mineral 2\% no inverno; Vertimec ${ }^{\circledR}$ $0,03 \%$ na brotação de novembro/dezembro até 2007). O itinerário técnico foi executado de acordo com as recomendações técnicas de KOLLER (1994; 2006).

No pomar orgânico, os tratos culturais foram realizados conforme as recomendações do Departamento Técnico da Cooperativa dos Citricultores Ecológicos do Vale do Caí -Ecocitrus. Consistiram em duas roçadas anuais da vegetação de cobertura do solo ao longo das 
linhas de laranjeiras. Nas entrelinhas, no período de outono/inverno, realizou-se o plantio de aveiapreta (Avena strigosa Schreber) e ervilhaca (Vicia sativa L.) e, no período de primavera-verão, a semeadura de feijão-miúdo (Vigna unguiculata (L.) Walp). Foram realizadas adubações anuais, de 2002 até 2005 no mês de maio, de $40 \mathrm{~m}^{3} \mathrm{ha}^{-1}$ de composto orgânico. Esta aplicação de composto foi suspensa devido à elevação do $\mathrm{pH}$ do solo. $\mathrm{O}$ manejo fitossanitário foi realizado com aplicação de caldas permitidas na agricultura orgânica (calda sulfocálcica 1\% (v/v) no período de agostosetembro; calda bordalesa $0,25 \%(\mathrm{p} / \mathrm{v})$ nos meses de setembro e março; calda bordalesa a $0,5 \%(\mathrm{p} / \mathrm{v})$ em novembro; Dipel ${ }^{\circledR}$ (Bacillus thuringiensis) a $0,15 \%(\mathrm{v} / \mathrm{v})$ até 2007).

A partir de cinco amostras por tratamento, em cada época avaliada (22/09/2012, 17/10/2012 e 11/10/2012), cada qual composta de 10 frutos, colhidos aleatoriamente de cinco plantas marcadas em cada pomar, determinou-se, em laboratório, as seguintes características físico-químicas: o teor de suco (TS), expresso em percentagem pela equação (massa do suco / massa total da amostra) x 100; teor de sólidos solúveis (SS), por refratometria e expressos em ${ }^{\circ}$ Brix; acidez titulável (AT), expressa em percentual de equivalente em ácido cítrico, determinada pela titulação de $6 \mathrm{~g}$ de suco com solução $0,1 \mathrm{~N}$ de $\mathrm{NaOH}$ até $\mathrm{pH}$ de 8,1 , calculada através da equação: $\mathrm{ATT}=[(\mathrm{ml}$ de $\mathrm{NaOH}) \times($ Normalidade do $\mathrm{NaOH}) \times 0,064 \times 100] /($ massa de suco $)$; relação $\mathrm{SS} /$ AT, calculada pela divisão dos valores de ${ }^{\circ} \mathrm{Brix}$ pelos valores de percentual de ácido cítrico (AOAC, 2000). A cor da casca (média de três medidas ao longo do eixo equatorial do fruto) foi obtida com auxílio de um Colorímetro (Konica/Minolta, CR400), obtendo-se as variáveis $L, a$ e $b$. Estas variáveis foram utilizadas para calcular o índice de cor da casca (ICC), que foi calculado do pela fórmula $(1000 \times a) /(L \times b)$ (JIMENEZ-CUESTA et al., 1981). Quando negativo, indica cores verdes e, quando positivo, cores alaranjadas, variando de $-20 \mathrm{a}+20$, em que o zero corresponde à cor amarela. Seguindo a indicação de SPÓSITO et. al.(2006), foram considerados aptos à comercialização os frutos com ICC $>2$.

Para as amostras dos testes de aceitação, também foram colhidos frutos aleatoriamente nos pomares, nas mesmas datas em que foram coletadas as amostras para as análises físico-químicas.

Os testes de aceitação foram realizados em sessões em laboratório, utilizando-se escala hedônica estruturada, de nove pontos, que expressam o grau de gostar ou desgostar do produto, na qual 1 equivale a desgostei muitíssimo, 5 nem gostei, nem desgostei e 9 equivale a gostei muitíssimo). $\mathrm{O}$ estudo foi realizado em cabines individuais sob luz branca, com um painel composto por 109 provadores (40 provadores, na primeira avaliação, 27 na segunda e 42 na terceira) de idade entre 18 a 64 anos, sendo todos funcionários, estudantes e professores de curso de graduação em engenharia de alimentos, nutrição e agronomia. As laranjas foram previamente lavadas e cortadas em quatro porções iguais, no sentido longitudinal e, para cada avaliador, foram oferecidas duas porções de laranjas, uma de cada tratamento, codificadas com algarismos de três dígitos aleatórios. As amostras foram servidas aos provadores em prato branco, com um copo com água à temperatura ambiente $\mathrm{e}$ uma ficha de avaliação sensorial. A ficha utilizada foi elaborada no sentido de identificar a percepção de qualidade dos três principais componentes de uma avaliação sensorial de laranjas: i) "atributo visual": aceitação da aparência da laranja, da casca e da cor dos frutos; ii) "atributo olfativo": odor; iii) "atributo gustativo": sabor, suculência, acidez e doçura, bem como a "aceitação global" da laranja: grau de gostar ou desgostar do produto de maneira global, adaptado de RÉVILLION et al. (2007).

$\mathrm{O}$ experimento foi conduzido em delineamento experimental completamente casualizado. Os atributos físicos e químicos dos frutos de cada tratamento foram submetidos a um diagnóstico de adequabilidade e posteriormente à análise de variância (ANOVA). Os resultados de aceitação das laranjas, em cada época avaliada, foram transformados por ordenamento dos dados e, após, foram submetidos à análise de variância multivariada (MANOVA) pelo teste de Wilks.

\section{RESULTADOS E DISCUSSÃO}

As características físicas e químicas dos frutos de ambos os sistemas de cultivo testados não apresentaram diferenças significativas na maioria dos atributos avaliados, sendo estas detectadas apenas no índice de cor da casca, na segunda época avaliada, e na acidez titulável e na relação SS/AT, na terceira época (Tabela 1). Diversos estudos apontam influência ou não do sistema de cultivo sobre os parâmetros qualitativos dos frutos. Por exemplo, ARRUDA et al. (2011) também não encontraram influência do sistema de cultivo orgânico e convencional em laranjas 'Valência' na maioria dos atributos qualitativos testados. 
Tabela 1 -Teor de suco (TS), teor de sólidos solúveis (SS), acidez titulável (AT), relação SS/AT e índice de cor da casca (ICC) de laranjas 'Valência' produzidas em sistema de cultivo orgânico e convencional, em setembro e outubro de 2011 e outubro de 2012 , em Montenegro-RS.

\begin{tabular}{|c|c|c|c|c|c|}
\hline & TS $(\%)$ & SS $\left({ }^{\circ} \mathrm{Brix}\right)$ & AT (\% ác. cítrico) & $\mathrm{SS} / \mathrm{AT}$ & ICC \\
\hline Orgânico & 0,53 & 10,02 & 1,02 & 9,78 & 7,18 \\
\hline Convencional & 0,55 & 9,84 & 1,06 & 9,27 & 6,38 \\
\hline $\mathrm{P}^{\mathrm{z}}$ & 0,30 & 0,65 & 0,47 & 0,40 & 0,48 \\
\hline \multirow[t]{2}{*}{ CV $(\%)$} & 4,86 & 6,03 & 8,15 & 9,70 & 24,96 \\
\hline & TS (\%) & SS $\left({ }^{\circ}\right.$ Brix $)$ & AT (\% ác. cítrico) & $\mathrm{SS} / \mathrm{AT}$ & $\mathrm{ICC}$ \\
\hline Orgânico & 0,52 & 10,76 & 0,88 & 12,23 & $8,30 *$ \\
\hline Convencional & 0,53 & 10,13 & 0,91 & 11,17 & 6,23 \\
\hline $\mathrm{P}$ & 0,46 & 0,24 & 0,37 & 0,04 & $<0,01$ \\
\hline \multirow[t]{2}{*}{$\mathrm{CV}(\%)$} & 4,03 & 7,60 & 5,18 & 5,88 & 11,35 \\
\hline & TS (\%) & SS $\left({ }^{\circ}\right.$ Brix $)$ & AT (\% ác. cítrico) & $\mathrm{SS} / \mathrm{AT}$ & $\mathrm{ICC}$ \\
\hline Orgânico & 0,52 & 9,52 & $0,81 *$ & $11,80 *$ & 4,41 \\
\hline Convencional & 0,50 & 10,35 & 1,03 & 10,10 & 4,75 \\
\hline $\mathrm{P}$ & 0,12 & 0,02 & $<0,01$ & $<0,01$ & 0,67 \\
\hline $\mathrm{CV}(\%)$ & 3,76 & 4,68 & 6,29 & 4,95 & 26,63 \\
\hline
\end{tabular}

${ }^{\mathrm{z}}$ Probabilidade de erro no teste $\mathrm{F} ; *$ Significativo quando $\mathrm{p}<0,01$.

LESTER et al. (2007) observaram que pomelos do pomar convencional apresentaram melhor coloração de casca e eram menos ácidos, enquanto os frutos do pomar orgânico apresentaram maior teor de sólidos solúveis.

Nas três épocas avaliadas, o teor de suco e a relação SS/AT das laranjas de ambos os tratamentos superaram os requisitos mínimos estabelecidos pelos padrões internacionais, definidos pela Organização para a Cooperação Econômica e Desenvolvimento (OECD, 2010). A casca dos frutos apresentou índice de cor da casca superior a 2, o que satisfaz o critério indicado por SPÓSITO et al. (2006) para comercialização in natura, e mostra que os frutos apresentavam uma coloração externa amareloalaranjado (Tabela 1).

A aceitação sensorial dos atributos dos frutos não diferiu entre os tratamentos nas três épocas avaliadas (Figura 1), demonstrando que há grande similaridade entre os frutos produzidos de ambos os sistemas de produção. Quanto à aparência, também não se observaram diferenças visuais (Figura 2).

Nas três épocas avaliadas, a aceitação foi satisfatória em todos os atributos avaliados (todos superiores à nota 6,5 - correspondente a $70 \%$ de aceitação). As diferenças observadas nos atributos físicos e químicos (Tabela 1) bem como por PETRY et al. (2012) não foram percebidas pelos avaliadores, provavelmente pela menor sensibilidade da percepção humana em relação aos indicadores físicos e químicos, o que deve ser estudado futuramente para que se possam determinar parâmetros físicos e químicos fiéis à aceitação pelo consumidor.

Em estudos de aceitação de maçãs produzidas em pomares convencionais e orgânicos, PECK et al.(2006) observaram que as maçãs do pomar orgânico foram de igual ou superior aceitação que as do convencional. DeELL \& PRANGE (1992) não observaram diferenças nos atributos sensoriais de duas cultivares de maçãs produzidas em sistemas de cultivo orgânico e convencional no momento da colheita e após quatro meses de armazenamento. Já LESTER et al. (2007) observaram que o suco de pomelos de pomar convencional, em relação aos de pomar orgânico, apresentaram melhor aceitação global pelos consumidores e maior doçura. Normalmente, a resposta quanto à preferência entre os produtos advindos de diferentes sistemas de cultivo variam, pois depende das características intrínsecas a cada espécie, das condições edafoclimáticas e do nível tecnológico usado em cada sistema. 


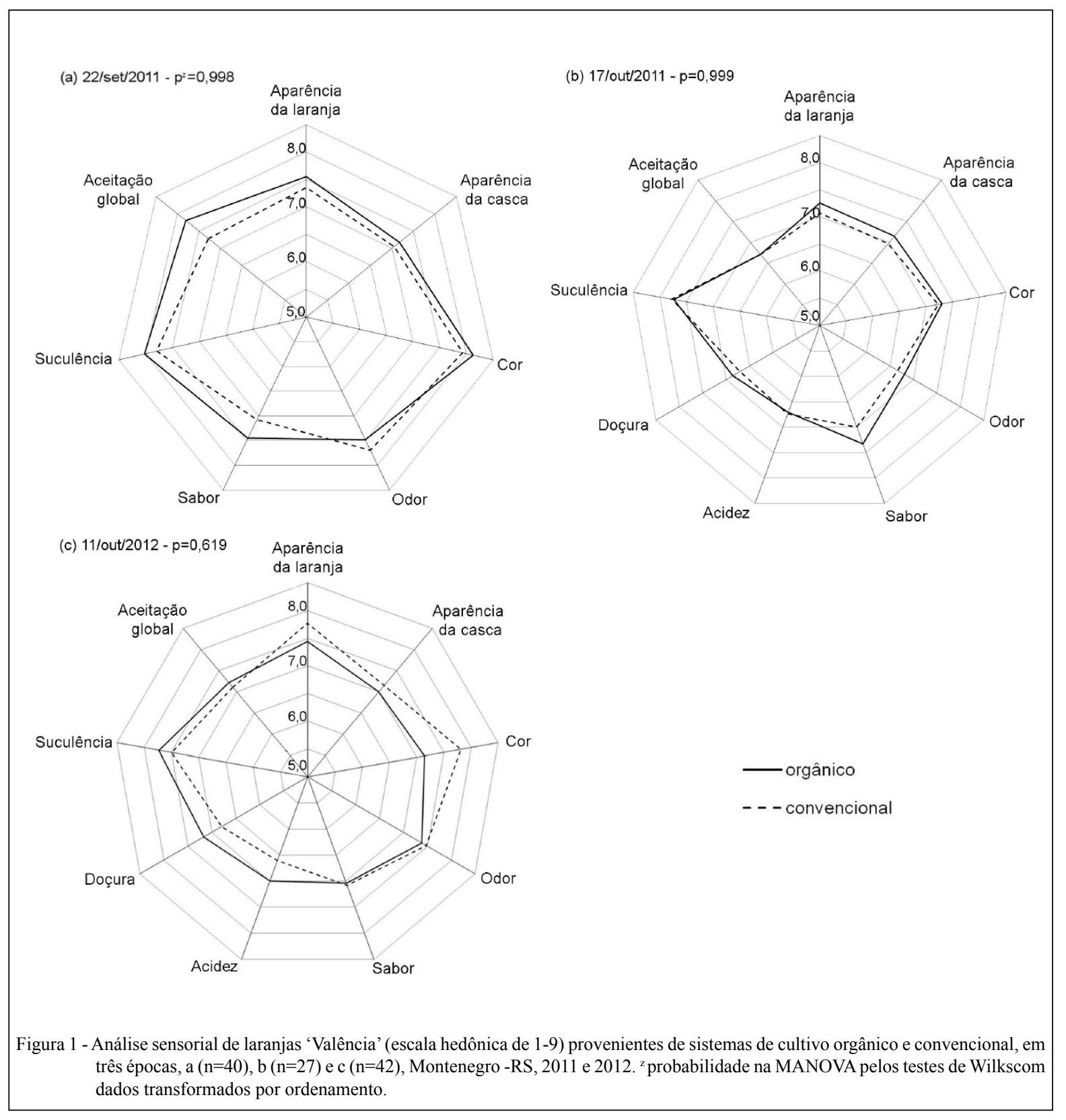

Adeptos de sistemas orgânicos de cultivo costumam afirmar que os frutos oriundos desses sistemas de cultivo apresentam qualidades superiores aos provenientes de sistemas convencionais (MAGKOS et al., 2006). Entretanto, eventuais diferenças existentes nos atributos qualitativos dos frutos provenientes de ambos os sistemas de cultivo não se manifestaram na análise sensorial nesta pesquisa, porque, provavelmente, foram imperceptíveis aos sentidos humanos. A utilização de avaliadores não treinados também pode explicar a não percepção de possíveis diferenças existentes.

\section{CONCLUSÃO}

Os sistemas de cultivo orgânico e convencional não influenciam nas características sensoriais de laranjas 'Valencia', para avaliadores não treinados.Há similaridade na qualidade física e química das laranjas produzidas em ambos os 


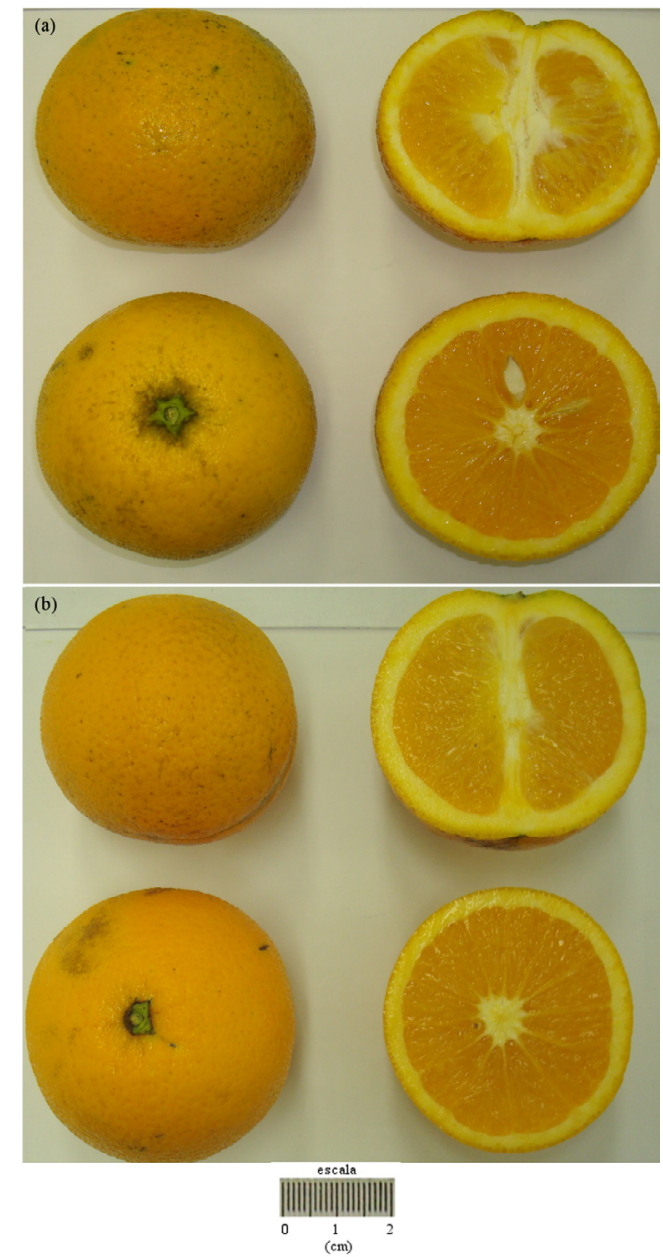

Figura 2 - Frutos produzidos sob sistema de cultivo convencional (a) e de sistema de cultivo orgânico (b) em Montenegro, RS, 2011. Foto: Henrique Belmonte Petry.

sistemas, o que pode ter refletido na não diferença de aceitação sensorial dos frutos.

\section{AGRADECIMENTOS}

À EMATER-RS e à Cooperativa Ecocitrus e seus membros, pelo apoio concedido na condução do experimento ao longo dos anos. Ao Ministério do Desenvolvimento Agrário e ao Conselho Nacional de Desenvolvimento Científico e Tecnológico $(\mathrm{CNPq})$, pelo apoio financeiro concedido por meio do edital 58/2010.Ao Eng. Agr. Bruno Casamali, estudante de Mestrado na Universidade da Florida, EUA, pela revisão da parte do texto escrito na língua inglesa.

\section{REFERÊNCIAS}

AGOSTINI, J.P. Manejo integrado de enfermedades de los frutales cítricos. In: SOZZI, G. (Ed. Lit.). Árboles frutales: ecofisiología, cultivo y aprovechamiento. Buenos Aires: Editorial Facultad de Agronomía, Universidad de Buenos Aires, 2007. p. 481-511.

AOAC (ASSOCIATION OF OFFICIAL ANALYTICAL CHEMISTS). Official methods of analysis of the Association of Official Analytical Chemists. 17. ed. Washington: AOAC International, 2000. 2200p.

ARRUDA, M.C. et al. Qualidade físico química de frutos de laranja Valência provenientes de cultivos orgânico e convencional. Citrus Research and Technology, Cordeirópolis, v.32, n.2, p.103-108, 2011. Disponível em: <http://revistalaranja.centrodecitricultura.br/index. php?pag=edicoes_revista\&edicaon=33>. Acesso em: 20 nov. 2013.

BERGAMASCHI, H. et al. Clima da Estação Experimental da UFRGS (e região de abrangência). Porto Alegre: UFRGS, 2003. 77 p.

BRASIL. Lei ${ }^{\circ} 10.831$, de 23 de dezembro de 2003. Dispõe sobre a agricultura orgânica e dá outras providências. Diário Oficial da União, Brasília, 24 de dezembro de 2003, Seção 1, p.8.

DeELL, J.R.; PRANGE, R.K. Postharvest quality and sensory attributes of organically and conventionally grown apples. Hortscience, Alexandria, v.27, n.10, p.1096-1099, 1992. Disponível em: <http://hortsci.ashspublications.org/ content/27/10/1096.full.pdf + html?sid=96082d62-477c-4de3bdb5-ae8b0667c38f>. Acesso em: 01 jul. 2013.

IBGE. Censo Agropecuário 2006. Rio de Janeiro, 2009. Disponível em: <http://www.ibge.gov.br/home/estatistica/economia/ agropecuaria/censoagro/brasil 2006/tab brasil/tab12.pdf>. Acesso em: 14 ago. 2013.

JIMENEZ-CUESTA, M. et al. Determination of a color index for Citrus fruit degreening. Proceedings of the International Society of Citriculture, Tokyo, v.2, p.750-753, 1981

KOLLER, O.C. Citricultura: laranja, limão e tangerina. Porto Alegre: Rigel, 1994. 446p.

KOLLER, O.C. (Org.). Citricultura: 1. Laranja: tecnologia de produção, pós-colheita, industrialização e comercialização. Porto Alegre: Cinco Continentes, 2006. 396p.

LESTER, G.E. et al. Organic vs conventionally grown Rio Red whole grapefruit and juice: Comparison of production inputs, market quality, consumer acceptance, and human health-bioactive compounds. Journal of Agricultural and Food Chemistry, Easton, v.55, n.11, p.4474-4480, 2007. Disponível em: <http:// pubs.acs.org/doi/pdf/10.1021/jf070901s $>$. Acesso em: 01 jul. 2013. doi: 10.1021/jf070901s.

MAGKOS, F. et al. Organic food: buying more safety or just peace of mind? A critical review of the literature. Critical Reviews in Food Science and Nutrition, Boca Raton, v.46, n.1, p.2356, 2006. Disponível em: <http://www.tandfonline.com/doi/ full/10.1080/10408690490911846\#.UdHhXGKTi6M>. Acesso em: 01 jul. 2013. doi: 10.1080/10408690490911846.

OECD. International standards for fruit and vegetables: citrus fruit. Paris, 2010. 244p.

PECK, G.M et al. Apple orchard productivity and fruit quality under organic, conventional and integrated management.

Ciência Rural, v.45, n.4, abr, 2015. 
Hortscience, Alexandria, v.41, n.1, p.99-107, 2006. Disponível em: $\quad<$ http://hortsci.ashspublications.org/content/41/1/99.full. pdf $>$. Acesso em: 01 jul. 2013.

PÉREZ-LÓPEZ, A.J. et al. Effects of organic farming on mineral contents and aroma composition of Clemenules mandarin juice. European Food Research and Technology, Berlin, v.225, n.2, p.255-260, 2007. Disponível em: <http://link.springer.com/ article/10.1007\%2Fs00217-006-0412-z/fulltext.html>. Acesso em: 08 ago. 2013. doi: 10.1007/s00217-006-0412-z.

PETRY, H.B. et al. Qualidade de laranjas 'Valência' produzidas sob sistema de cultivo orgânico e convencional. Revista Brasileira de Fruticultura, Jaboticabal, v.34, n.1, p.167174, 2012. Disponível em: <http://www.scielo.br/scielo. php?script $=$ sci_arttext\&pid=S0100-29452012000100023\&lng $=$ en $\& n r m=i s o>$. Acesso em: 01 jul. 2013. doi: 10.1590/S010029452012000100023

RÉVILLION, J.P.P. et al. Qualidade sensorial de vinhos tintos finos do Rio Grande do Sul comparados aos importados da Argentina e Chile. Ciência e Tecnologia de Alimentos, Campinas, v.27, n.1, p.177-180, 2007. Disponível em: <http://www.scielo.br/pdf/cta/ v27n1/30.pdf $>$. Acesso em: 08 ago. 2013. doi: 10.1590/S010120612007000100031.
SABA, A.; MESSINA, F. Attitudes towards organic foods and risk/benefit perception associated with pesticides. Food Quality and Preference, Barking, v.14, n.8, p.637-645, 2003 Disponível em: <http://www.sciencedirect.com/science/article/ pii/S095032930200188X>. Acesso em: 01 jul. 2013. doi: S095032930200188X.

SPÓSITO, M.B. etal. Determinação do índice de cor mínimo necessário para a colheita de laranja doce Valência a ser submetida ao processo de desverdecimento. Laranja, Cordeirópolis, v.27, n.2, p.373-379, 2006. Disponível em: $<\mathrm{http} / / /$ revistalaranja.centrodecitricultura.br/index. php?pag=edicoes_revista\&edicaon=15>. Acesso em: 01 jul. 2013

STRECK, E.V. et al. Solos do Rio Grande do Sul. 2.ed. rev. e ampl. Porto Alegre: Emater/RS, 2008. 222p.

TEIXEIRA, L.J.Q. et al. Hábitos de consumo de frutas entre estudantes da Universidade Federal de Viçosa. Ceres, Viçosa, v.53, n.307, p.366-373, 2006. Disponível em: $<$ http://www.ceres.ufv.br/ ceres/revistas/V53N307P05206.pdf>. Acesso em: 01 jul. 2013.

UNCTAD (UNITED NATIONS CONFERENCE ON TRADE AND DEVELOPMENT).Citrus fruit: quality. Disponível em: $<$ http://r0.unctad.org/infocomm/anglais/orange/quality.htm $>$. Acesso em: 01 jul. 2013. 


\section{ERRATA}

Artigo “Avaliação física e química e aceitação pelo consumidor de laranjas 'Valência', produzidas sob sistema de cultivo orgânico e convencional” publicado no periódico Ciência Rural v45n4 páginas 619-625 sem a inclusão do capítulo COMITÊ DE ÉTICA HUMANA:

leia-se:

\section{“COMITÊ DE ÉTICA HUMANA}

Nós autores do artigo intitulado "Avaliação física e química e aceitação pelo consumidor de laranjas 'Valência', produzidas sob sistema de cultivo orgânico e convencional" declaramos, para os devidos fins, que o projeto que deu origem aos dados do mesmo não foi submetido para avaliação ao Comitê de Ética da UFRGS, mas estamos cientes do conteúdo da resolução № 466 , de 12 de dezembro de 2012 do Conselho Nacional de Saúde <http://conselho.saude.gov.br/resolucoes/2012/Reso466.pdf> caso envolva humanos.

Desta forma, os autores assumem total responsabilidade pelos dados apresentados e estão disponíveis para possíveis questionamentos, caso venham a ser requeridos pelos órgãos competentes."

Para a versão correta, acesse:

http://www.scielo.br/pdf/cr/v45n4/1678-4596-cr-45-04-00619.pdf 